\title{
BURDEN OF DISEASE AND SOCIETAL COSTS DUE TO MENINGOCOCCAL DISEASE IN DENMARK - A NATIONAL REGISTER BASED STUDY
}

Lars Østergaard ${ }^{1}$, Nina Gustafsson², Sandra Elkjær Stallknecht², Mette Skovdal ${ }^{3}$, Peter Bo Poulsen ${ }^{3}$

1. Aarhus University Hospital, Aarhus, Denmark; 2. Incentive, Holte, Denmark; 3. Pfizer Denmark, Ballerup, Denmark

\section{Background}

Despite being preventable by vaccine, meningococcal disease $(\mathrm{MD})$ remains a substantial global public health burden. Incidence rates of MD are highest in children, adolescents and young adults. The incidence has decreased in the past decades, although still being severe to the affected cases each year. In Europe 1.1 MD case per 100,000 (2004) and 0.7 MD case per 100,000 in Denmark (2015) were affected.

However, detailed information on the burden and the associated societal costs of MD especially in Europe - is limited. The objective was therefore to estimate the societal costs of meningococcal disease (MD) and the burden of disease in Denmark.

\section{Methods}

The study was designed as a retrospective study based on national Danish registries covering all Danes. Incident patients with a primary diagnosis of MD (ICD-8 code 036 (from 1980 to 1993) or ICD-10 code A39 (from 1994 to 2015)) were identified in the National Patient Register and matched with two controls using direct matching on age, gender and level of education. Siblings constituted a secondary control population (Figure 1).

With a societal perspective, costs related to healthcare services in hospitals and primary care, prescription medicine, municipality homecare services, and lost production were included. All costs were inflated to 2015 prices and converted to US\$ (USD 1= DKK 6.73).

Average actual costs were defined as average costs per person in a given year before/after MD diagnosis. Future costs due to MD were simulated with

projections from 2017 to 2060 . Burden of disease was assessed by analyses on mortality, sequelae a posteriori and followup on labour market status.

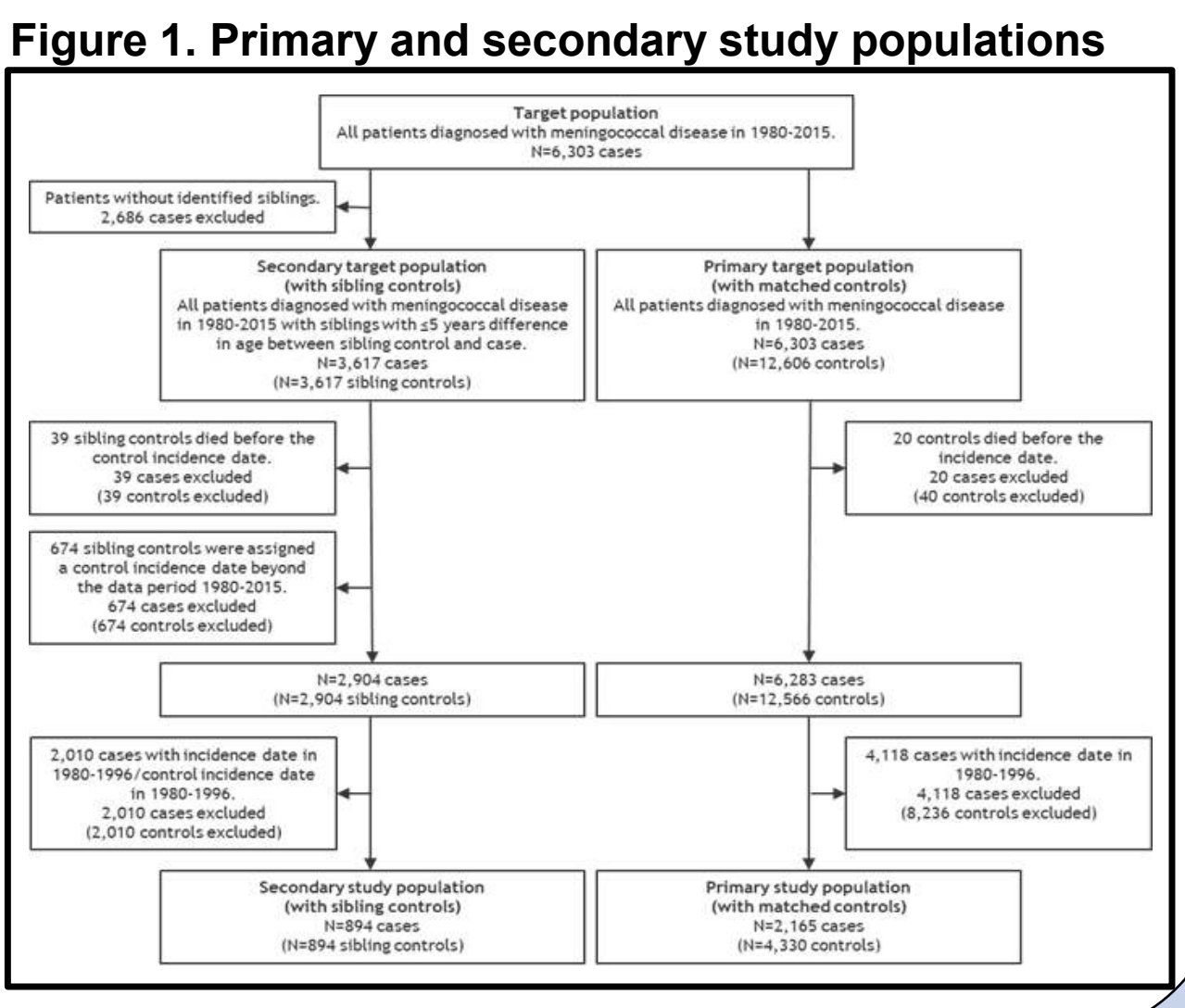

\section{Conflict of interest}

This study was supported by Pfizer Denmark. Incentive was a paid vendor of Pfizer Denmark. Sandra Elkjær Stallknecht and Nina Gustafsson are employees at Incentive. Lars Jørgen Østergaard was a paid clinical consultant of Pfizer Denmark. Peter Bo Poulsen and Mette Skovdal are employees at Pfizer Denmark.

\section{Results}

Patients diagnosed with MD have more sequelae, lower employment rates in the first year after diagnosis, and the mortality is significantly affected in the period immediate after the diagnosis. Actual health care costs are high (Table 1). The differences in costs between cases and controls are most significant in the baseline year (case: $\$ 3,834$; control: $\$ 1,742$ ) and the first year after diagnosis (case: $\$ 16,641$; control: $\$ 1,426$ ). Most plausibly the increased costs in the baseline year is explained by more prior comorbidities among cases compared to controls. Similar results were found with sibling controls.

Table 1. Average actual costs per patient in the baseline year (year 0 ) and the first five years after MD diagnosis among patients diagnosed in 1997-2015, and their matched controls/siblings (US\$ 2015 prices)

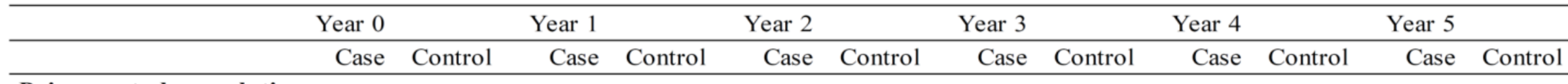
\begin{tabular}{lllllllllllll}
\hline Primary study population & & & & & & & & & & & & \\
$\mathrm{N}$ & 2,165 & 4,330 & 2,165 & 4,330 & 1,913 & 3,826 & 1,828 & 3,656 & 1,768 & 3,536 & 1,743 & 3,486 \\
\hline
\end{tabular} \begin{tabular}{lrrrrrrrrrrrr}
$\mathrm{N}$ & 2,165 & 4,330 & 2,165 & 4,330 & 1,913 & 3,826 & 1,828 & 3,656 & 1,768 & 3,536 & 1,743 & 3,486 \\
\hline Primary sector costs & 329 & 260 & 350 & 249 & 268 & 217 & 246 & 214 & 214 & 212 & 214 & 196 \\
Outpatient costs & 503 & 267 & 1,096 & 249 & 470 & 263 & 387 & 229 & 348 & 238 & 333 & 259
\end{tabular} $\begin{array}{lrrrrrrrrrrrr}\text { Outpatient costs } & 503 & 267 & 1,096 & 249 & 470 & 263 & 387 & 229 & 348 & 238 & 333 & 259 \\ \text { Hospital admission costs } & 2,591 & 864 & 14,817 & 659 & 1,090 & 536 & 824 & 568 & 598 & 493 & 549 & 413\end{array}$ Medicine costs Home care costs Grand total $\begin{array}{rrrrrrrrrrrr}2,591 & 864 & 14,817 & 659 & 1,090 & 536 & 824 & 568 & 598 & 493 & 549 & 413 \\ 221 & 172 & 197 & 159 & 185 & 146 & 174 & 148 & 157 & 136 & 149 & 133 \\ 190 & 179 & 182 & 110 & 91 & 86 & 40 & 26 & 16 & 2 & 41 & 2\end{array}$ Secondary study populatio Primary sector costs Outpatient costs Hospital admissi
Medicine costs Medicine costs Grame care costs

\begin{tabular}{rrrrrrrrrrrr}
894 & 894 & 894 & 894 & 799 & 799 & 766 & 766 & 742 & 742 & 732 & 732 \\
\hline 268 & 212 & 288 & 199 & 221 & 190 & 198 & 189 & 192 & 188 & 192 & 186 \\
371 & 174 & 979 & 153 & 413 & 150 & 307 & 163 & 365 & 175 & 320 & 162 \\
1,608 & 549 & 13,225 & 330 & 756 & 332 & 569 & 256 & 454 & 253 & 308 & 210 \\
110 & 81 & 115 & 95 & 113 & 96 & 113 & 102 & 112 & 93 & 108 & 96 \\
0 & 0 & 3 & 0 & 0 & 0 & 0 & 0 & 0 & 0 & 0 & 0 \\
2,357 & 1,016 & 14,610 & 777 & 1,504 & 768 & 1,186 & 709 & 1,124 & 709 & 928 & 654 \\
\hline
\end{tabular}

As the Danish population grow in coming decades, the future costs of MD are estimated to increase. Based on the study data, a conservative estimate projects annual costs up to US\$ 1.5-1.7 million in the period 2017-2060. A less conservative and higher estimate, incl. losses due to premature death, projects annual costs between US\$ 4.1-4.6 million.

\section{Conclusions}

- The societal costs and burden of meningococcal disease are substantial with hospital admission costs and production loss as main cost drivers.

- Costs are highest from the age of 14 and onwards.

- It could be reasonable that any kind of preventive activity were to target this or other age-groups using this evidence on the impact of the disease. 